\title{
The Distribution of Cloud to Ground Lightning Strike Intensities and Associated Magnetic Inductance Fields near the Kennedy Space Center
}

\author{
Lee Burns. \\ Raytheon, Huntsville, AL, 35805 \\ and \\ Ryan Decker ${ }^{\dagger}$ \\ NASA, Marshall Space Flight Center, AL, 35812
}

\begin{abstract}
Lightning strike location and peak current are monitored operationally in the Kennedy Space Center (KSC)/Cape Canaveral Air Force Station (CCAFS) area by the Cloud to Ground Lightning Surveillance System (CGLSS). The present study compiles ten years worth of CGLSS data into a database of near strikes. Using shuttle launch platform LP39A as a convenient central point, all strikes recorded within a 20 -mile radius for the period of record (POR) from January 1, 1993 to December 31, 2002 were included in the subset database. Histograms and cumulative probability curves are produced for both strike intensity (peak current, in $\mathbf{k A}$ ) and the corresponding magnetic inductance fields (in $\mathbf{A} / \mathbf{m}$ ). Results for the full POR have application to launch operations lightning monitoring and post-strike test procedures.
\end{abstract}

$\mathrm{I}_{\mathrm{p}} \quad=$ peak current $(\mathrm{kA})$

MI = Magnetic Inductance $(\mathrm{A} / \mathrm{m})$

$\mathrm{d}=$ Great Circle Distance (m)

\section{Nomenclature}

\section{Introduction}

T ightning activity in the Kennedy Space Center (KSC) area is monitored continuously by the Cloud to Ground LLightning Surveillance System (CGLSS), which has been in operation since 1990. The system consists of six independent and geographically dispersed lightning detection and direction sensors, a centralized strike position analyzer, and a networked data display. The position analyzer and the data display are collocated in the Range Operations Command Center (ROCC) at Cape Canaveral Air Force Station. The locations of the six sensors, along with the ROCC, are shown in Fig. 1. Also shown in Fig. 1 is the shuttle launch pad LP39A, which is used as a convenient point of reference for KSC. The current results are then strictly applicable to Shuttle pad and launch operations. However, due to general consistency of the geographical distributions of strikes in the overall region, other areas of KSC should expect similar distributions near strikes over time. Hence the current study is also of interest to other current or future vehicles that do or will launch out of KSC. A ring of $32 \mathrm{~km}$ radius, centered on LP39A, is also shown in Fig. 1. Only lightning strikes that occurred within this circle are considered in the following analysis. A detailed description of the CGLSS hardware, system capabilities, and operational limitations can be found in the CGLSS Instrumentation Handbook ${ }^{1}$, which is available online at http://wwwsdd.fsl.noaa.gov/RSA/cglss/CGLSS inst handbook.pdf.

The collected CGLSS data is operationally archived for the ongoing period from January 1, 1990 to the present. For each lightning strike detected, the time of occurrence, the latitude and longitude of the occurrence, and the peak

\footnotetext{
- Senior Physics Engineer, MSFC/EV13, Marshall Space Flight Center, Alabama, 35812

† Aerospace Technologist, Flight Vehicle Atmospheric Environments, Natural Environments Branch/EV13, Marshall Space Flight Center, Alabama, 35812
} 
current, $I_{p}$, are determined by the system. The data is stored in tabular ASCII format, and is available online at ftp://trmm.ksc.nasa.gov/midds/LLP/. For convenience, LP39A was used as a specific positional surrogate for KSC. From the latitude and longitude of the strike, and knowing the latitude and longitude of LP39A, the great circle distance, d, between the strike and LP39A can be computed. After obtaining all data for the 10-year Period of Record (POR, January 1, 1993 to December 31, 2002), a database subset was developed consisting of all lightning strikes that occurred within $32 \mathrm{~km}$ of KSC. Then the magnetic inductance (MI) field that would be produced at LP39A as a result of the strike can be computed from the simple diagnostic equation

$$
M I=I_{p} / 2 \pi d
$$

The original inclusive database contains in excess of 1.5 million lightning strikes. The subset database contains 173039 individual strikes.

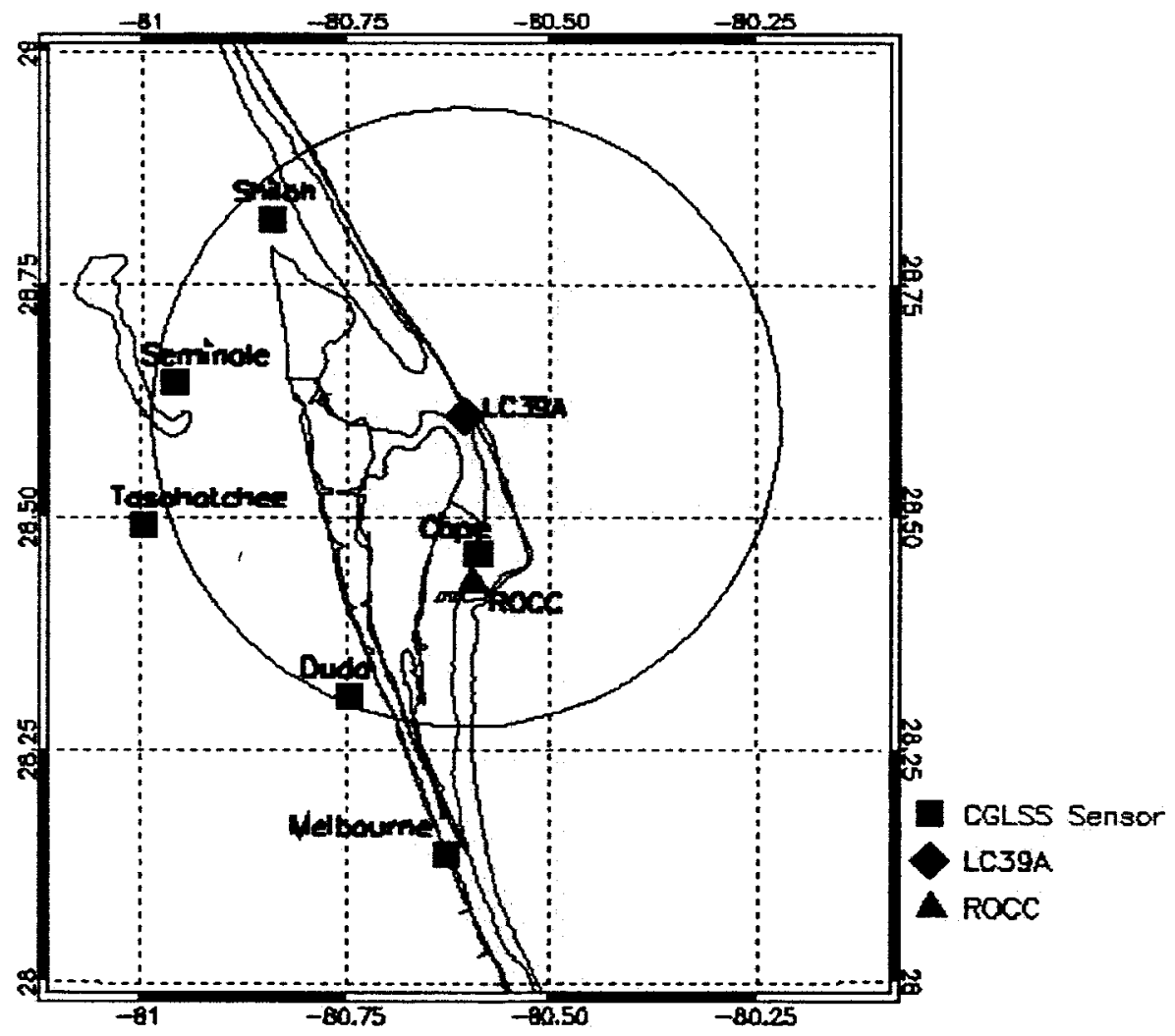

Figure 1. Map of the KSC area showing locations of the CGLSS sensors and the ROCC, along with LP39A. Also shown is a circle of 32-km radius, centered on LP39A.

\section{Results}

Histograms of lightning strike intensities (in $\mathrm{kA}$ ) were produced for various time partitions of the POR. Figure 2 shows histograms for each individual year, highlighting the interannual variability. Also shown in Fig. 2 is a histogram representing the distribution of all strikes in the POR, and one representing the mean annual distribution. Figure 3 shows the distributions of strike intensities by month. For example, the histogram in panel (a) compiles the strikes that occurred in the 10 January months in the POR. Figure 4 shows the distributions of MI fields produced at LP39A by strikes grouped by year, as in Fig. 2. Figure 5 shows the monthly distributions of MI fields at LP39A 
corresponding to the strike intensity histograms in Fig. 3. In the figures, the y-axis is scaled logarithmically to enhance detail at low occurrence values.

As is evident from the figures, there is significant variability in both the monthly and interannual strike distributions. It was expected to find such variability in the monthly values, as the general weather patterns at KSC are known to follow fairly ordered cycles with large seasonal amplitudes. However, the degree of interannual variability was unexpected. Table 1 presents the total number of strikes for each month and each year in the POR. The table also gives the annual, and monthly mean strike counts, as well as the standard deviations.

Table 2 gives the magnitude of the strike intensities that correspond to various cumulative probability limits. For example, from the table, on an annual basis, there is a $95 \%$ probability that a given strike will have an intensity magnitude less than or equal to $58.3 \mathrm{kA}$. Table 3 gives the MI fields that correspond to various cumulative probability limits.
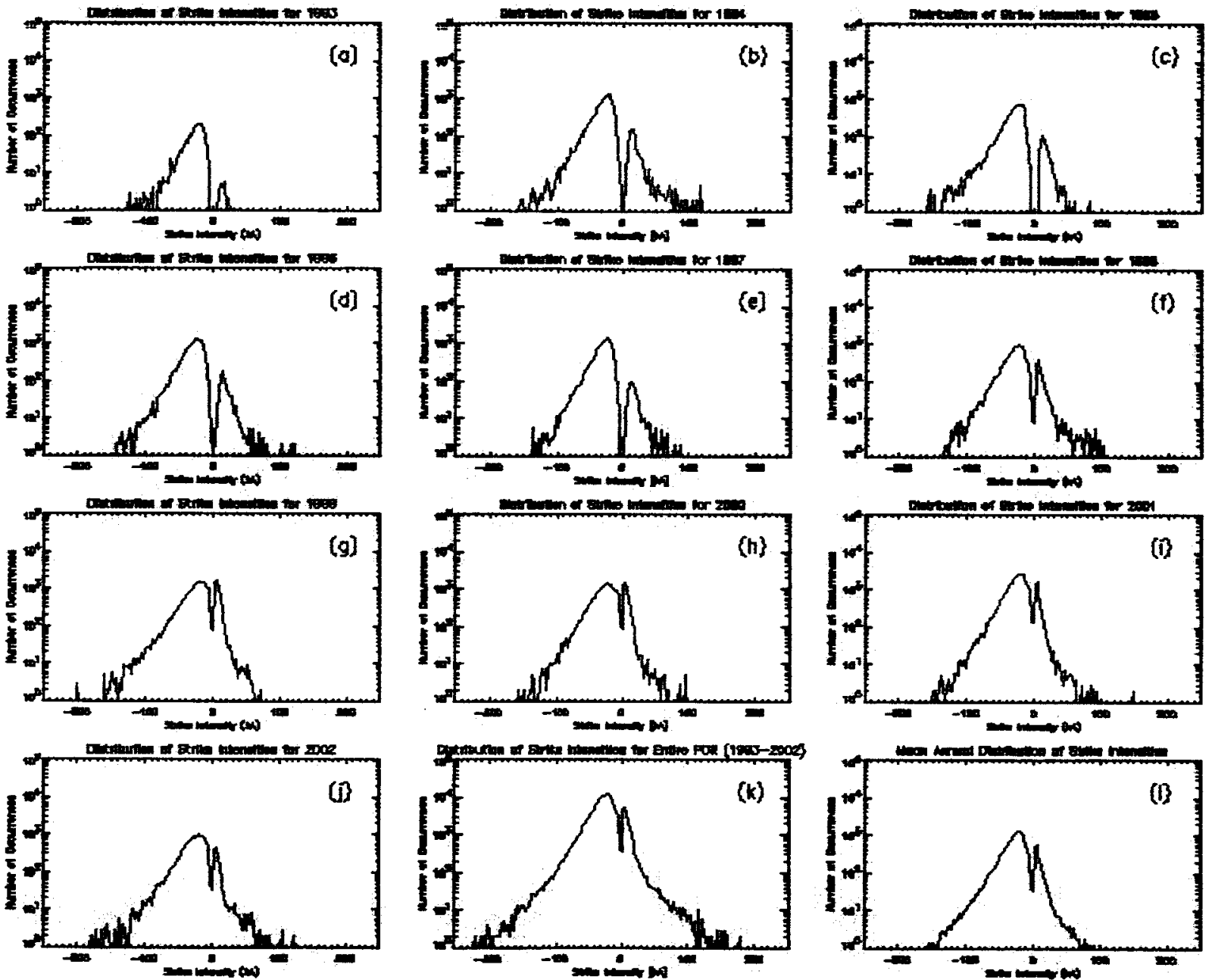

Figure 2. Histograms of lightning strike intensities for all strikes occurring within $32 \mathrm{~km}$ of LP39A for (a) 1993, (b) 1994, (c) 1995, (d) 1996, (e) 1997, (f) 1998, (g) 1999, (h) 2000, (i) 2001, (j) 2002, (k) the entire POR, and (l) mean annual distribution. 

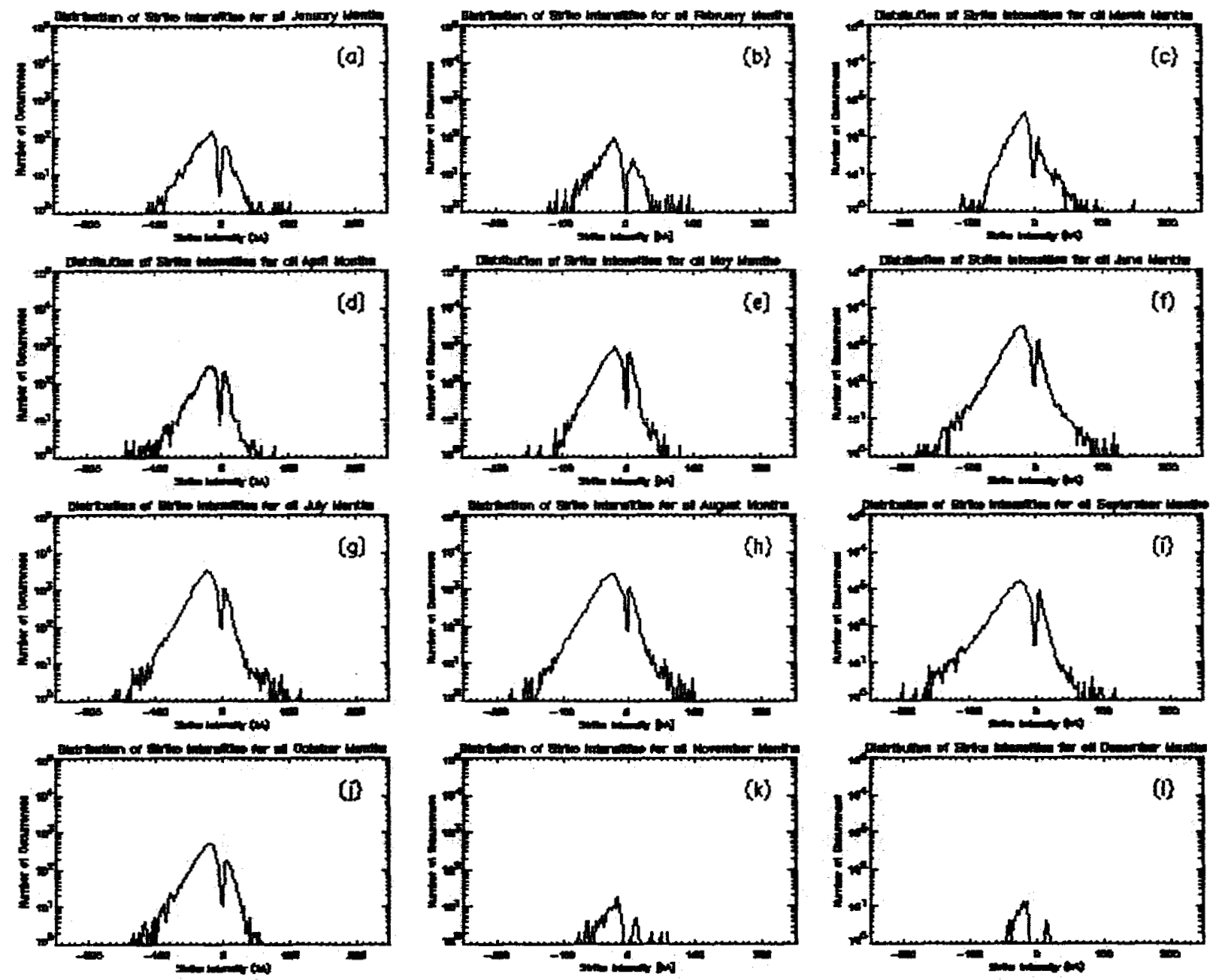

Figure 3. Histograms of lightning strike intensities for all strikes occurring within $32 \mathrm{~km}$ of LP39A for (a) all January months in the POR, (b) February months, (c) March months, (d) April months, (e) May months, (f) June months, (g) July months, (h) August months, (i) September months, (j) October months, (k) November months, and (l) December months. 

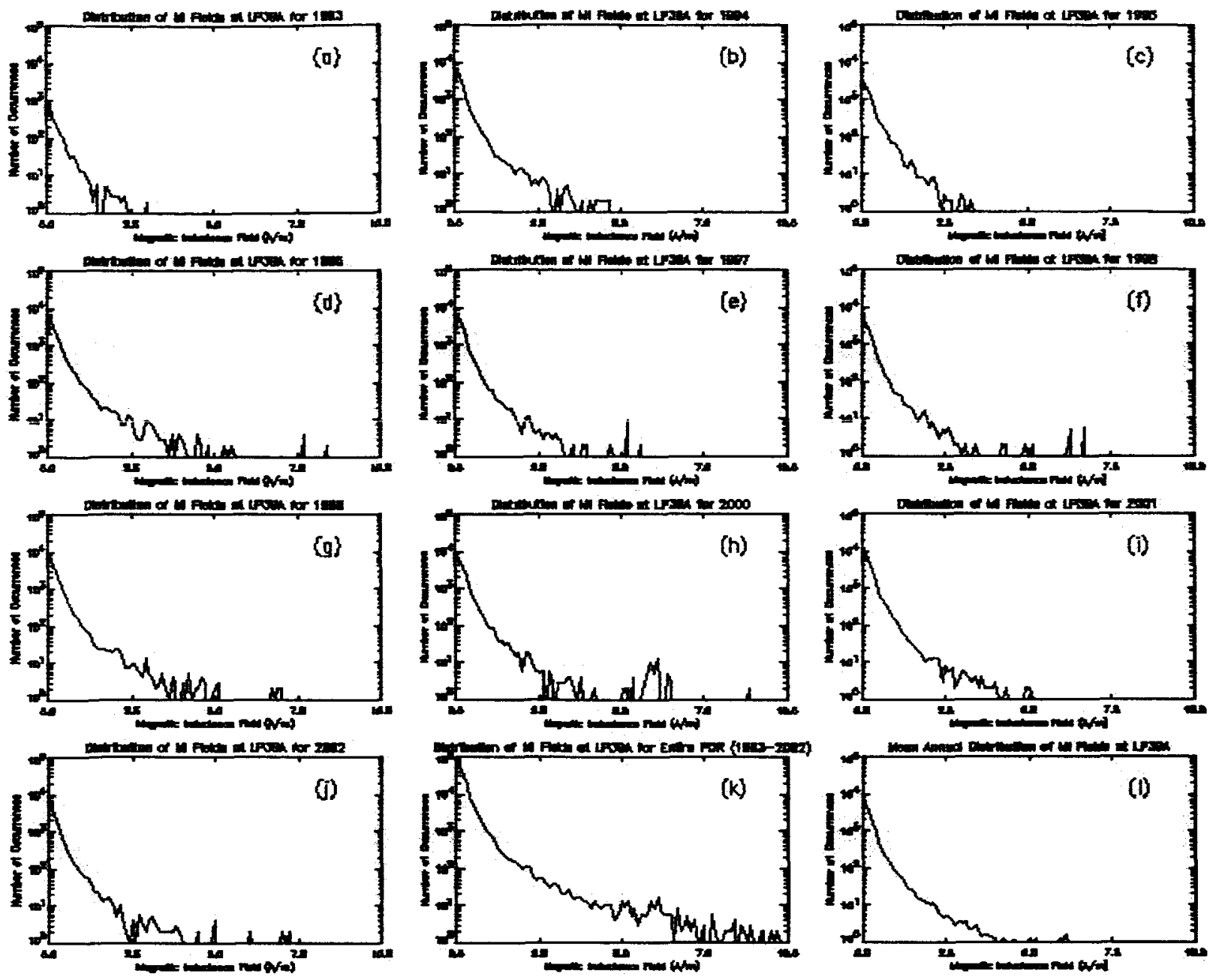

Figure 4. Histograms of MI fields produced at LP39A, for all strikes occurring within $32 \mathrm{~km}$ for (a) 1993, (b) 1994, (c) 1995, (d) 1996, (e) 1997, (f) 1998, (g) 1999, (h) 2000, (i) 2001, (j) 2002, (k) the entire POR, and (l) mean annual distribution. 

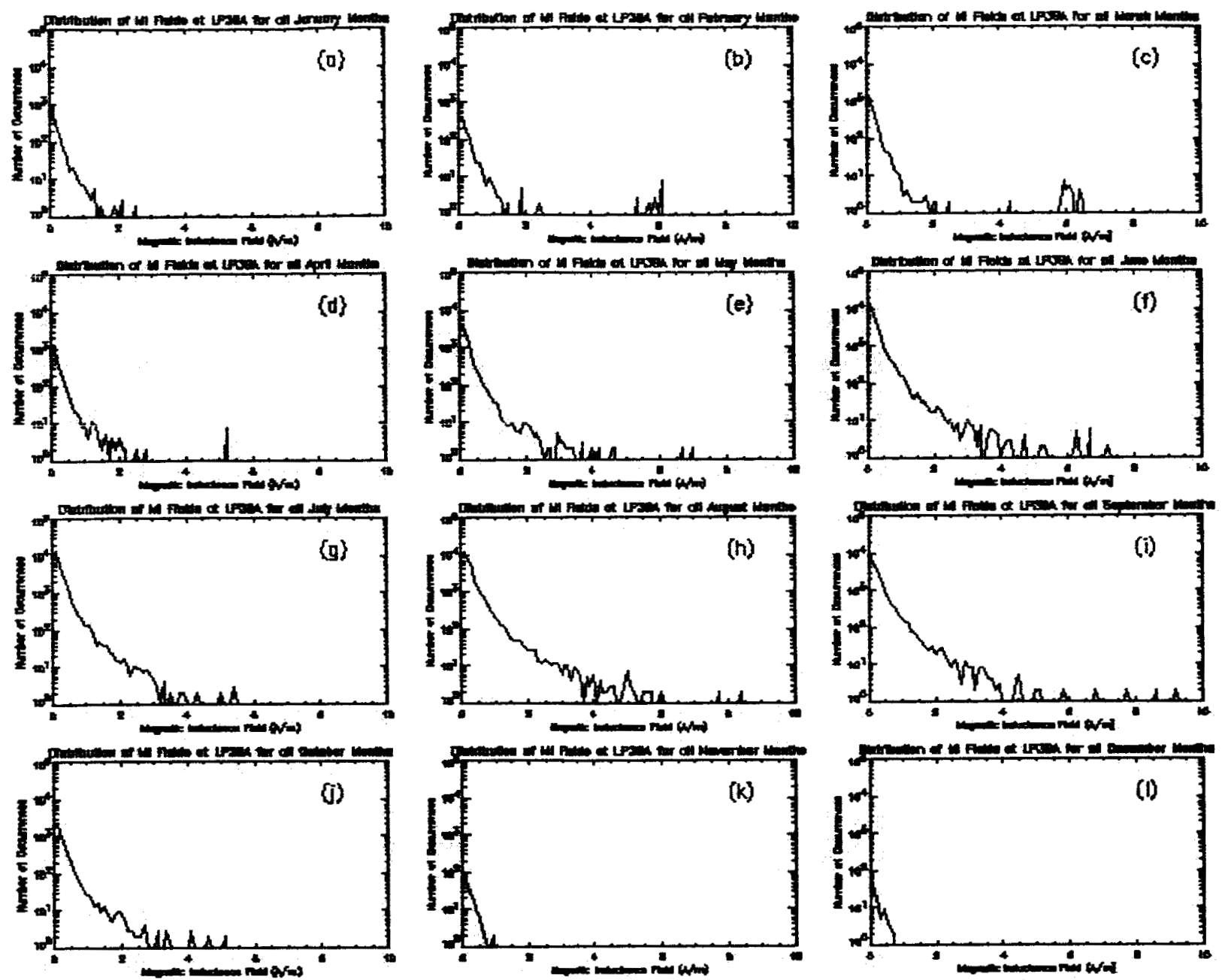

Figure 5. Histograms of MI fields produced at LP39A, for all strikes occurring within $32 \mathrm{~km}$ for (a) all January months in the POR, (b) February months, (c) March months, (d) April months, (e) May months, (f) June months, (g) July months, (h) August months, (i) September months, (j) October months, (k) November months, and (l) December months. 
Table 1. Strike Count Statistics for Yearly and Monthly Time Periods.

\begin{tabular}{|c|c|c|c|c|c|c|c|c|c|c|c|c|c|}
\hline & 1993 & 1994 & 1995 & 1996 & 1997 & 1998 & 1999 & 2000 & 2001 & 2002 & Totals & $\bar{\mu}$ & $\bar{\sigma}$ \\
\hline Jan & 0 & 2 & 131 & 164 & 594 & 99 & 869 & 0 & 8 & 39 & 1906 & 191 & 298 \\
\hline Feb & 0 & 1 & 1 & 0 & 0 & 672 & 341 & 24 & 1 & 113 & 1153 & 115 & 223 \\
\hline Mar & 0 & 132 & 98 & 30 & 0 & 423 & 268 & 365 & 1594 & 1302 & 4212 & 421 & 565 \\
\hline Apr & 0 & 753 & 0 & 79 & 157 & 2 & 650 & 877 & 857 & 267 & 3642 & 364 & 375 \\
\hline May & 0 & 956 & 0 & 0 & 1197 & 675 & 6623 & 658 & 1073 & 102 & 11284 & 1128 & 1987 \\
\hline Jun & 1580 & 2518 & 600 & 5864 & 2034 & 1538 & 4120 & 3218 & 13761 & 3197 & 38430 & 3843 & 3791 \\
\hline Jul & 17 & 3083 & 6011 & 1200 & 5051 & 3204 & 2643 & 8363 & 8510 & 2337 & 40419 & 4042 & 2873 \\
\hline Aug & 0 & 1847 & 1898 & 4015 & 5538 & 4588 & 5829 & 2858 & 6950 & 5711 & 39234 & 3923 & 2211 \\
\hline Sep & 317 & 1904 & 290 & 4218 & 292 & 2373 & 4053 & 7590 & 3367 & 1026 & 25430 & 2543 & 2335 \\
\hline Oct & 1000 & 2967 & 0 & 22 & 228 & 380 & 510 & 7 & 1509 & 1226 & 7009 & 701 & 952 \\
\hline Nov & 0 & 0 & 1 & 0 & 50 & 0 & 8 & 32 & 77 & 13 & 181 & 18 & 27 \\
\hline Dec & 0 & 2 & 0 & 0 & 107 & 0 & 0 & 0 & 0 & 7 & 116 & 12 & 34 \\
\hline Totals & 2074 & 14165 & 9030 & 15592 & 15248 & 13954 & 25914 & 23992 & 37707 & 15340 & 173016 & & \\
\hline $\boldsymbol{\mu}$ & 173 & 1180 & 753 & 1299 & 1271 & 1163 & 2160 & 1999 & 3142 & 1278 & & & \\
\hline $\boldsymbol{\sigma}$ & 454 & 1223 & 1743 & 2122 & 1975 & 1487 & 2410 & 3007 & 4366 & 1733 & & & \\
\hline
\end{tabular}

Table 2. Strike Intensity Magnitudes for Various Cumulative Probability Limits.

\begin{tabular}{|c|c|}
\hline Probability & Strike Intensity (kA) \\
\hline 0.5 & 22.4 \\
\hline 0.9 & 47.4 \\
\hline 0.95 & 58.3 \\
\hline 0.98 & 74.4 \\
\hline 0.99 & 88.3 \\
\hline 0.999 & 150.0 \\
\hline
\end{tabular}

Table 3. Magnetic Inductance Fields at LP39A for Various Cumulative Probability Limits

\begin{tabular}{|c|c|}
\hline Probability & MI Field (A/m) \\
\hline 0.5 & 0.173 \\
\hline 0.9 & 0.495 \\
\hline 0.95 & 0.712 \\
\hline 0.98 & 1.13 \\
\hline 0.99 & 1.62 \\
\hline 0.999 & 5.99 \\
\hline
\end{tabular}




\section{Summary}

The distribution of cloud to ground lightning strikes at KSC shows significant variability on both a monthly and interannual basis. For the POR, the greatest monthly strike count occurred in June 2001. Many individual months had no recorded strikes within a 20-mile radius of LP39A, with these occurring typically in the winter months. On a monthly strike rate basis, July had the greatest average number of strikes with 4042 per month, while December had the lowest average with only 12 strikes per month. On an interannual basis, 2001 had the greatest average strike rate with an average of 3142 strikes per month, while 1993 had the lowest average strike rate with only 173 strikes per month. Cumulative probabilities values have been calculated to show what limiting strike magnitudes and what magnetic inductance fields at LP39A correspond to various probability limits.

The subset data base derived in this study has applicability towards addressing to a wide range of engineering design and operations support issues. For example, characterizing the lightning environment at KSC is an important step in the design process for flight hardware that will operate out of the KSC spaceport. Understanding the lightning environment is also important for the design and operation of adverse-effect mitigation systems that protect sensitive electronics on launch vehicles, payloads, and in ground-based launch support facilities. This type of data can also be applied to the diagnosis of particular case study events. For example, the data set has been used as part of the Columbia accident investigation by giving the locations and strike magnitudes of all cloud to ground lightning strikes that occurred near LP39A during the time that the STS-107 vehicle was on the pad, between rollout and launch

\section{Reference}

${ }^{1}$ CSR Corporation, Systems Analysis Department, 2002: Eastern Range Instrumentation Handbook. CSR Corporation, Orlando, FL 